\title{
Has the era of dual-gated myocardial perfusion SPECT and PET arrived?
}

\author{
Koichi Okuda, PhD, ${ }^{\mathrm{a}}$ and Kenichi Nakajima, $\mathrm{MD}, \mathrm{PhD}^{\mathrm{b}}$ \\ a Department of Physics, Kanazawa Medical University, Kahoku, Ishikawa, Japan \\ b Department of Nuclear Medicine, Kanazawa University Hospital, Kanazawa, Japan
}

Received Aug 30, 2018; accepted Aug 31, 2018

doi:10.1007/s12350-018-1439-9

\section{See related article, pp. 634-647}

Many studies have investigated how to eliminate image blurring caused by cardiac and respiratory motions in cardiac single-photon emission computed tomography (SPECT) and positron emission tomography (PET) images. Cardiac electrocardiographic (ECG)gated SPECT and PET are established methods with which to monitor the beating heart, which allows evaluation of cardiac functions such as left ventricular ejection fraction (LVEF), cardiac volume, and the contraction indices. Consequently, an algorithm was created to generate images that are free of cardiac motion, and it has been clinically applied. ${ }^{1}$ Image blurring caused by cardiac motion was eliminated while myocardial count density was maintained. By contrast, some medical devices can monitor and measure abdominal and thoracic movements during respiration, ${ }^{2}$ and a data-driven algorithm to detect respiratory motion is also available. ${ }^{3}$ Therefore, clinical dual-gated myocardial perfusion SPECT and PET image acquisitions could become routine considering these technological advancements.

However, since organ displacement cannot be accurately measured due to cardiac and respiratory motions in clinical patients and precise organ movements cannot be reproduced in an acrylic phantom, algorithms and software for detecting respiratory and cardiac motions are difficult to validate. A mathematical digital phantom is a powerful simulation tool that can

\footnotetext{
Reprint requests: Koichi Okuda, PhD, Department of Physics, Kanazawa Medical University, 1-1 Daigaku, Uchinada, Kahoku, Ishikawa, 920-0293, Japan; okuda@nmd.m.kanazawa-u.ac.jp J Nucl Cardiol 2020;27:648-650.

$1071-3581 / \$ 34.00$

Copyright (C) 2018 American Society of Nuclear Cardiology.
}

evaluate the effects of cardiac and respiratory motions on the quality of SPECT and PET images. A 4D extended cardiac-torso (XCAT) anthropomorphic digital phantom based on a realistic model of patient anatomy is available from the Duke University. ${ }^{4}$ Cardiac and respiratory motions can be simulated in this phantom based on gated X-ray computed tomography data, and amplitudes and timing for each motion can be mathematically controlled. We acquired virtual myocardial perfusion SPECT images using the 4D XCAT digital phantom through simulating medical imaging nuclear detectors (SIMIND) Monte Carlo software ${ }^{5}$ to authenticate a novel program that can evaluate ECG-gated myocardial perfusion SPECT data. ${ }^{6}$ Figure 1 shows an example of normal myocardial perfusion images derived from a clinical patient and a digital phantom. The image quality of the digital phantom was essentially equivalent to that of the clinical patient. This is because not only cardiac ${ }^{99 \mathrm{~m}} \mathrm{Tc}$ activity but also hepatic, pulmonary, and background activities in the digital phantom were considered in that comparison (Figure 1A, B). Moreover, cardiac and other organ movements were also represented. Polar maps created out of data generated from the digital phantom and the patients corresponded in terms of ECGgated SPECT functions such as perfusion at end-diastolic and end-systolic timings, wall motion, and wall thickening (Figure 1C).

The study by Zhang and colleagues published in this issue of the Journal of Nuclear Cardiology evaluated respiratory-gated methods to reduce motion-induced blurring in myocardial perfusion SPECT images. ${ }^{7}$ Alternative time- and amplitude-based respiratory curves can be used to acquire respiratory-gated images from list-mode datasets. That cited study compared respiratory amplitude (AG)-, equal count (EG)-, and equal time- or phase (TG)-gated images acquired from 10 digital phantom models and clinical patients. Acquired counts are typically limited in myocardial perfusion SPECT, but appropriate count statistics can be 
a

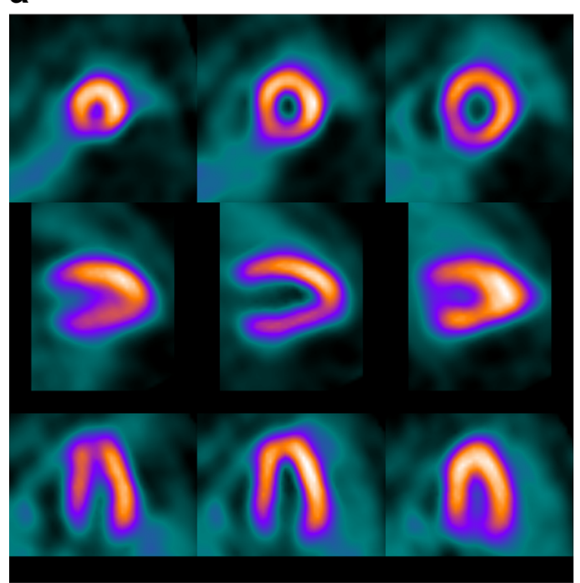

b

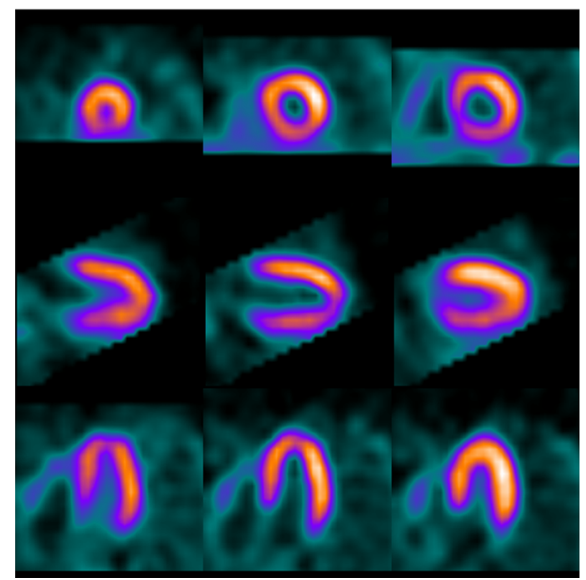

c
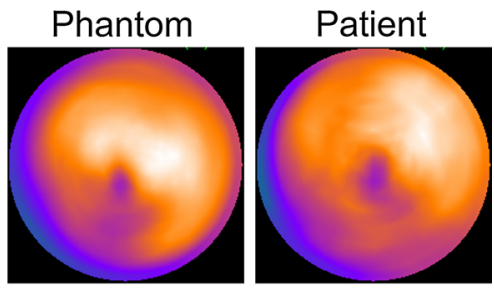

Perfusion

at ED
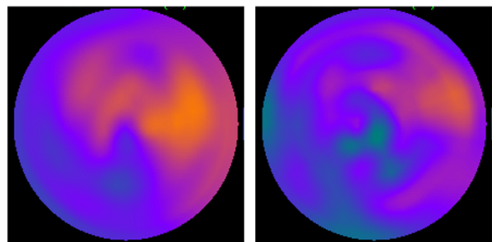

Perfusion


Wall

motion
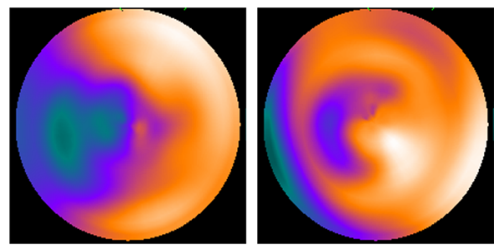

Wall thickening

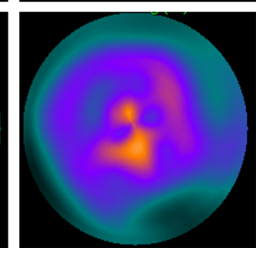

Figure 1. Examples of ${ }^{99 \mathrm{~m}} \mathrm{Tc}$ normal myocardial perfusion distribution in a digital phantom and a clinical patient. Apical, mid, and basal short-axis, and vertical, and horizontal long-axis displays of myocardial perfusion distribution derived from a male digital phantom (A) and a 72-year-old man with a low likelihood of coronary artery disease (B). Ejection fractions of patient and digital phantoms were $62 \%$ and $61 \%$, respectively. Polar maps of perfusion in ECG-non-gated images, ED, ES, wall motion, and thickening (C). ECG, electrocardiographic; $E D$, end-diastolic; and $E S$, endsystolic.

obtained by image registration processing in which each respiratory bin is registered to a reference bin. Zhang et al. fit respiratory bins to the reference bin using an affine and b-spline nonrigid registration method. Respiratory motion was reduced more in $A G$ and EG than in TG. Cardiac and respiratory motions will require compensation to precisely determine myocardial perfusion distribution. Compensating dual-gated PET myocardial perfusion images with cardiac and respiratory motions has improved image resolution, contrast, and contrastto-noise ratios. ${ }^{8}$ However, further investigation of routine clinical dual-gated myocardial perfusion SPECT images are required, since very few studies have addressed this matter. ${ }^{9}$
In addition to image-registration processing, respiratory-gated imaging using a cadmium zinc telluride (CZT) gamma camera might be promising, ${ }^{10}$ because this type of camera has high-sensitivity detectors that can monitor respiratory motion even during short acquisition periods. ${ }^{3}$ However, myocardial perfusion distribution differs according to the structure of CZT and Anger gamma cameras, as well as the scan position and time. ${ }^{11}$ Therefore, the quality of respiratory-compensated myocardial perfusion images needs to be confirmed for each instrument. Moreover, the applicability of dual-gated myocardial perfusion imaging will require multicenter validation studies using digital phantoms and clinical patients. Normal databases for the 
calculation of summed stress/rest/difference scores might be updated for dual-gated imaging, and the effects of dual-gated imaging on normal values for LVEF, volume, and contraction indices should be also investigated.

Clinical applications of cardiac and respiratorygated myocardial perfusion SPECT and PET imaging methods are just emerging. When ECG gating was introduced during the 1990s, it was rapidly accepted for clinical practice, which resulted in more quantitative functional evaluation. The need for ECG gating is not in any doubt. Respiratory gating is theoretically desirable to reduce artifacts caused by complicated cardiac movements, but the technology will need to improve before it can become clinically applicable. A digital phantom is suitable for dual-gated myocardial perfusion studies to confirm the performance of a novel or improved compensation algorithm for cardiac and respiratory motions. Since digital phantoms and Monte Carlo simulation codes are widely available, the accuracy of cardiac and respiratory-gated SPECT and PET imaging can continuously improve. Perhaps dual-gated imaging will become commonplace in clinical practice within 10 years, but quality, reliability, and practicality will require enhancement in the meantime.

\section{Acknowledgments}

This study was partly funded by the JSPS KAKENHI Grants (Numbers 26861022). The authors appreciate the editorial assistance of Norma Foster.

\section{Disclosures}

The authors have no conflicts of interest to declare.

\section{References}

1. Slomka PJ, Nishina H, Berman DS, Kang X, Akincioglu C, Friedman JD, et al. "Motion-frozen" display and quantification of myocardial perfusion. J Nucl Med 2004;45:1128-34.

2. Hyun MC, Gerlach J, Rubeaux M, Slomka PJ. Technical consideration for dual ECG/respiratory-gated cardiac PET imaging. $\mathrm{J}$ Nucl Cardiol 2017;24:1246-52.

3. Ko CL, Wu YW, Cheng MF, Yen RF, Wu WC, Tzen KY. Datadriven respiratory motion tracking and compensation in CZT cameras: A comprehensive analysis of phantom and human images. J Nucl Cardiol 2015;22:308-18.

4. Segars WP, Sturgeon G, Mendonca S, Grimes J, Tsui BM. 4D XCAT phantom for multimodality imaging research. Med Phys 2010;37:4902-15

5. Ljungberg M, Strand S-E. A Monte Carlo program for the simulation of scintillation camera characteristics. Comput Methods Progr Biomed 1989;29:257-72.

6. Okuda K, Nakajima K, Kikuchi A, Onoguchi M, Hashimoto M. Cardiac and respiratory motion-induced artifact in myocardial perfusion SPECT: 4D digital anthropomorphic phantom study. Ann Nucl Cardiol 2017;3:88-93.

7. Zhang D, Pretorius PH, Ghaly M, Zhang Q, King MA, Mok GSP. Evaluation of different respiratory gating schemes for cardiac SPECT. J Nucl Cardiol 2018. https://doi.org/10.1007/s12350-0181392-7.

8. Slomka PJ, Rubeaux M, Le Meunier L, Dey D, Lazewatsky JL, Pan T, et al. Dual-gated motion-frozen cardiac PET with Flurpiridaz F 18. J Nucl Med 2015;56:1876-81.

9. Kortelainen MJ, Koivumaki TM, Vauhkonen MJ, Hedman MK, Karkkainen STJ, Nino Quintero J, et al. Respiratory motion reduction with a dual gating approach in myocardial perfusion SPECT: Effect on left ventricular functional parameters. J Nucl Cardiol 2017. https://doi.org/10.1007/s12350-017-0844-9.

10. Daou D, Sabbah R, Coaguila C, Boulahdour H. Feasibility of datadriven cardiac respiratory motion correction of myocardial perfusion CZT SPECT: A pilot study. J Nucl Cardiol 2017;24:1598607

11. Oddstig J, Martinsson E, Jogi J, Engblom H, Hindorf C. Differences in attenuation pattern in myocardial SPECT between CZT and conventional gamma cameras. J Nucl Cardiol 2018. 